\title{
Mean QA Interval
}

National Cancer Institute

\section{Source}

National Cancer Institute. Mean QA Interval. NCI Thesaurus. Code C120922.

The calculated average time period between the recording of a Q wave on ECG and the onset of the aortic blood pressure pulse as determined over a number of cardiac cycles during a given interval of time. 